\title{
Factores modificantes del movimiento dentario ortodóncico
}

\section{Modifiers factors of orthodontic tooth movement}

\author{
Vargas del Valle $\mathrm{P}^{*}$, Piñeiro Becerra $M S^{*}$, Palomino Montenegro $\mathrm{H}^{*}$,**, \\ Torres-Quintana MA***
}

\begin{abstract}
RESUMEN
El fenómeno de la remodelación ósea es fundamental tanto para ortodoncia como para la ortopedia dentofacial. Esta revisión clínica es sobre la relación de la actividad ósea desencadenada por las fuerzas ortodóncicas con diversos factores, como factores propios de los pacientes, moléculas producidas por tejidos enfermos, o drogas y nutrientes consumidas regularmente por los pacientes, los que pueden alcanzar los tejidos periodontales mecánicamente estresados por las fuerzas ortodóncicas a través de la circulación sanguínea, interactuando así con células blanco que producen la remodelación ósea necesaria para el movimiento dentario ortodóncico. El efecto combinado de estas fuerzas mecánicas con alguno de estos agentes pueden ser inhibitorio, aditivo o sinérgico. El objetivo de esta revisión es describir los mecanismos de acción y los efectos de algunas de las drogas y otros factores sobre la remodelación del tejido óseo y el movimiento dentario ortodóncico.
\end{abstract}

Palabras clave: Movimiento dentario, ortodoncia, factores modificantes.

\section{SUMMARY}

Bone remodeling is fundamental to orthodontics and dentofacial orthopedics. This clinically oriented overview relate bone responses to orthodontic forces with many factors. Molecules produced in various diseased tissues, or drugs and nutrients consumed regularly by patients, can reach the mechanically stressed paradental tissues through the circulation, and interact with local target cells. The combined effect of mechanical forces and one or more of these agents may be inhibitory, additive or synergistic. The objective of this review is to outline the mechanisms of action and effects of some commonly used drugs and other factors on tissue remodeling and orthodontic tooth movement.

Key words: Tooth movements, orthodontics, modifiers factors.

Fecha de recepción: 17 de septiembre de 2009.

Aceptado para publicación: 22 de septiembre de 2009.

* Universidad Andrés Bello. Facultad de Odontología. Dpto. de Niño y adolescente.

** Universidad Andrés Bello. Facultad de Odontología. Dpto. de Niño y adolescente; y Universidad de Chile. Facultad de Odontología. Dpto. de Niño y Ortopedia Dentomaxilar.

*** Universidad de Chile. Facultad de Odontología. Dpto. de Patología.

Vargas del Valle P, Piñeiro Becerra MS, Palomino Montenegro H, Torres-Quintana MA. Factores modificantes del movimiento dentario ortodóncico. Av. Odontoestomatol 2010; 26 (1): 45-53.

\section{INTRODUCCIÓN}

El tratamiento ortodóncico implica el uso y control de fuerzas que producen remodelación de tejidos dentarios y peridentarios, incluyendo la pulpa dental, ligamento periodontal, hueso alveolar y encía. Estos tejidos expresan grandes cambios microscópicos y macroscópicos, al ser expuestos a distin- 
tos grados de magnitud, frecuencia y duración de fuerzas.

En la respuesta tisular al estímulo ortodóncico, varios tejidos se ven contemporáneamente afectados, esto implica una serie de reacciones celulares y la intervención de múltiples factores que pueden modificar el éxito y las expectativas de la terapia. La mecánica propia al tratamiento, esto es, la magnitud y dirección de las fuerzas aplicadas, deben ser cuidadosamente evaluadas y manejadas por el clínico. La edad, la presencia de enfermedades óseas y el consumo de fármacos que puedan afectar el metabolismo óseo, son factores del paciente que pueden alterar la efectividad del tratamiento. Finalmente, están todos aquellos factores externos, que han sido ampliamente investigados tanto en modelos animales como en estudios clínicos con el fin de favorecer o limitar ciertos movimientos dentarios. El objetivo de esta revisión es describir algunos de estos factores y su efecto sobre la remodelación de tejidos y el movimiento dentario ortodóncico (MDO), así como los mecanismos de acción y los efectos de algunas drogas y estímulos mecánicos.

\section{FACTORES PROPIOS DEL PACIENTE EN MOVIMIENTO DENTARIO ORTODÓNCICO}

En la planificación y desarrollo del tratamiento ortodóncico se deben considerar las características particulares de cada paciente, porque pueden alterar de manera significativa el tiempo y los objetivos del tratamiento.

\section{Edad}

Por mucho tiempo se ha creído que el tratamiento ortodóncico en adultos toma más tiempo que en niños y adolescentes, pero la relación entre la edad y el MDO aun no ha sido completamente comprendida (1). Se ha observado que los tejidos peridentarios del adulto presentan características diferentes a la de los niños o adolescentes (2). Al aumentar la edad, la actividad celular disminuye, y los tejidos se hacen más ricos en colágeno, afectando la respuesta de los tejidos adultos a las fuerzas ortodóncica $(3,4)$.
El niño, está en una etapa proliferativa, y presenta un hueso alveolar esponjoso, con espacios medulares grandes y numerosos, el flujo vascular es abundante, y presenta un máximo potencial de remodelación. El ligamento periodontal en el niño presenta una alta tasa de renovación fibrilar, las fibras colágenas son más finas y hay mayor número de células; esto hace que los tejidos periodontales en individuos jóvenes reaccionen más rápidamente a la carga ortodóncica, a diferencia de la respuesta de los tejidos periodontales del adulto (5). Esto se debe a los cambios fisiológicos que sufre el tejido periodontal en el adulto, donde el hueso alveolar está menos vascularizado y los espacios medulares adquieren más tejido adiposo. Del mismo modo, la tasa de renovación celular en el ligamento periodontal, es menor en el adulto, la situación es más estable, y las fibras colágenas son más gruesas lo que retrasa la respuesta proliferativa ante la aplicación de fuerzas ortodóncicas (6).

Ren y cols. (4), estudiaron las fases del MDO en ratas adultas y jóvenes, encontrando que la fase inicial del movimiento dentario experimental en animales adultos es más lenta que en el animales jóvenes, pero esta diferencia entre los grupos de edades no se encontró en la fase linear del movimiento. Se ha descrito además, que en el adulto, se forman más fácilmente las zonas hialinas en el lado de presión, las cuales pueden limitar temporalmente el MDO (7).

\section{Fármacos}

El paciente en tratamiento ortodóncico, puede estar consumiendo una gran variedad de medicamentos, pero aquellos que pueden alcanzar el tejido óseo mecánicamente estresado, a través de la circulación sanguínea, interactuando con las células "blanco" locales, son los que pueden afectar principalmente el tratamiento ortodóncico. La combinación del efecto mecánico de las fuerzas y algunos de estos agentes puede resultar en una inhibición o estimulación para el movimiento dentario (8). Dentro de este grupo están los antiinflamatorios no esteroidales (AINEs) y los bifosfonatos inhibiendo el MDO y los corticosteroides estimulándolo.

El grupo de fármacos más comúnmente utilizados durante la terapia ortodóncica son los AINEs, utiliza- 
dos para control del dolor tras la aplicación de las fuerzas sobre las piezas dentarias. El efecto antiinflamatorio de este grupo es resultado del bloqueo de la síntesis de prostaglandinas a partir del ácido araquidónico, mediante la inhibición de la enzima ciclooxigenasa $(9,10)$. Estudios clínicos y en animales, han identificado el rol de las prostaglandinas en el proceso de reabsorción ósea, determinando que tienen una acción directa en el aumento del número y tamaño de osteoclastos y en la estimulación de su actividad resortiva $(8,9)$.

Es por esto que el uso de AINEs para control de dolor en ortodoncia, ha sido cuestionado en los últimos años, porque no sólo tendrían un efecto positivo en el manejo del dolor tras las activaciones ortodóncicas, pero también podrían afectar la secuencia del movimiento dentario, mediante la inhibición, o al menos la disminución de la relación entre la inflamación y el proceso de reabsorción ósea (8), disminuyendo el rango de movimiento dentario (12). Los estudios destinados para aclarar esta relación han tenido resultados dispares. En 1992, Wong y cols. (13), concluyeron que la administración de ácido acetilsalicílico a cobayas no tenía mayor efecto sobre el movimiento dentario, en cambio estudios realizados por Arias y Márquez-Orozco (10), mostraron que el rango de movimiento dentario en ratas en el grupo que habían recibido aspirina e ibuprofeno, era menor que el de los controles, y del grupo que recibió paracetamol. También se ha sometido ha estudio la administración de bloqueadores selectivos de la COX2 , los que han reemplazado el uso de los AINEs convencionales en el control del dolor postoperatorio. Gameiro y cols. (14), establecieron que la administración de Celecoxib no afectaba el número de osteoclastos, pero al parecer disminuye la efectividad de éstos, lo que explicaría la inhibición del movimiento dentario en los individuos tratados con este fármaco.

La mayoría de los estudios demuestran la efectividad de los AINEs en el control del dolor, pero también establecen que de algún modo estos pueden afectar la efectividad del movimiento dentario $(8,15)$, por esto hoy las líneas de investigación tratan de determinar cual sería el antiinflamatorio con menor impacto en el remodelado óseo, y las últimas publicaciones señalan al paracetamol como una buena alternativa.
El Paracetamol es una de las drogas más usadas para el seguro y efectivo control del dolor, éste actúa disminuyendo los productos de la ciclooxigenasa, preferentemente en el sistema nerviosos central (16), sin alterar significativamente la secreción periférica de PGs es así que estudios experimentales en animales han concluido que el uso de paracetamol no alteraría el remodelado óseo, es decir no tendría efecto sobre el rango de movimiento dentario $(10,17)$.

El creciente uso de glucocorticoides para el tratamiento de condiciones médicas, tales como artritis, alergias, alteraciones renales, de colágeno y otras, ha hecho que tanto clínicos como investigadores se interesen en entender los mecanismos mediante los cuales estos medicamentos podrían alterar el metabolismo óseo (18). Los corticoides están relacionados con el control de carbohidratos, grasa y el metabolismo de proteínas, pero también tienen propiedades antiinflamatorias. También tienen una participación en la fisiología ósea, aunque su rol no se ha definido claramente (19). Los efectos secundarios a largo plazo de estas terapias radican en alteraciones en la mineralización de los tejidos duros y en la cicatrización, además de alteraciones en la condrogénesis y osteogénesis, pérdida ósea y osteoporosis (18). Estudios en ratas con osteoporosis inducida, han establecido que el rango de movimiento es mayor cuando se administran corticoides en forma crónica, pero que este movimiento es menos estable, por la mala calidad y escasa neoformación ósea. En administraciones agudas el remodelado parece ser más lento. Clínicamente los resultados sugieren que se puede tratar ortodóncicamente a pacientes con terapias de corticoides, pero se deben tener ciertas consideraciones. En casos de pacientes sometidos a terapia de corticoides por un tiempo corto, es recomendable posponer la mecánica ortodóncica hasta que deje de recibir el fármaco. En casos de corticoterapias crónicas, al ser limitada la formación ósea, se recomienda realizar una mecánica ortodóncica con fuerzas reducidas y controlar al paciente más seguido (20).

Otro grupo de fármacos que pueden tener efecto sobre el remodelado óseo son los bifosfonatos, usados para el tratamiento de la osteoporosis. Esta es una enfermedad muy común en mujeres adultas, 
condición caracterizada por una pérdida de resistencia y masa ósea. La mayoría de las drogas usadas para su tratamiento son antirreabsorción ósea, lo que retrasa la fase destructiva (reabsorción) del remodelado óseo (8). Los bifosfonatos (como el alendronato o risedronato, entre otros) son fármacos con alta afinidad por el calcio y se dirigen a áreas de remodelado óseo, inhiben el metabolismo osteoclástico y reducen el número de estas células. Esto indicaría que los bifosfonatos pueden inhibir el movimiento dentario y retardar el tratamiento ortodóncico $(9,21)$. Además otros estudios han establecido que el uso de bifosfonatos aumenta el riesgo de osteonecrosis, asociado a procedimientos quirúrgicos complementarios al tratamiento ortodóncico, como extracciones o instalación de microimplantes (21). Es de gran importancia considerar que la vida media de estos fármacos es extremadamente larga, incluso mayor a los 10 años. Generalmente se presume que este grupo de drogas se mantienen inactivas retenidas en el tejido óseo hasta que la droga activa es liberada por el proceso normal de remodelado óseo (22).

\section{AGENTES EXTERNOS EN EL MOVIMIENTO DENTARIO ORTODÓNCICO}

Los grandes avances en el conocimiento molecular, han permitido a los investigadores buscar alternativas para acelerar o inhibir el movimiento en ciertas piezas dentarias, con el objetivo de obtener la máxima eficiencia en la terapia ortodóncica, para esto se han utilizados diversas sustancias químicas, y algunos agentes físicos, como láser, campos electromagnéticos, y ultrasonido.

\section{Factores limitantes del movimiento dentario ortodóncico}

Con el objeto de limitar el movimiento ortodóncico, en casos que requieren de anclaje máximo, o para evitar las recidivas post-tratamiento, se han realizado estudios para poder contar con alternativas a los dispositivos mecánicos intra o extraorales que utilizamos en la clínica en la actualidad.

Estudios experimentales con aplicaciones tópicas de bifosfonatos, han establecido que estas drogas son capaces de inhibir fuertemente la reabsorción ósea asociada al movimiento dentario (23).

También se ha estudiado la administración local de echistatina y del péptido arginina-glicina-ácido aspártico (RGD), en ratas, para limitar el movimiento dentario por inhibición de la reabsorción ósea y la reabsorción radicular. Éstos son agentes inhibidores de integrinas o también llamadas desintegrinas, las que se asocian directamente a la respuesta celular antes cargas mecánicas. La echistatina, una desintegrina derivada del veneno de serpiente, ha demostrado ser potente inhibidor de la reabsorción ósea in vivo, su función se atribuye a la disminución de la función osteoclástica por inhibición de la integrina alpha(v)beta(3), lo que reduce su capacidad de migración y de mantención de la zona del sellado durante el proceso de formación de lagunas de reabsorción, mientras que el RGD disminuye el número de osteoclastos (24).

Kansaki y cols. (25), en observaciones in vitro estableció que las células del ligamento periodontal, ante fuerzas mecánicas de compresión, inducen osteoclastogénesis a través de la regulación de la expresión de ligando del receptor activador del factor nuclear kappa B (RANKL) vía síntesis de PGE2. En el año 2004, investigaron la acción del gen osteoprotegerina (OPG) en este proceso, demostrando que la transferencia del gen OPG a los tejidos periodontales inhibe la acción del RANKL limitando el movimiento dentario ortodóncico (26). Son necesarios más estudios preclínicos con estos fármacos o agentes, para ser utilizados clínicamente.

\section{Factores estimulantes del movimiento dentario ortodóncico}

\section{Agentes químicos}

Desde el punto de vista del paciente, el tiempo total de tratamiento es uno de los factores más importantes, es por esto que muchos estudios se han dedicado a analizar distintos métodos para acelerar el MDO, tales como la inyección de ciertas drogas o la utilización de algunos agentes físicos (27).

Al conocerse el importante rol de las prostaglandinas (PGs) en las distintas secuencias que llevan al MDO, 
se vio en ellas la posibilidad de incrementar el rango de movimiento. De este modo se desarrollaron varios estudios, en ratas y otros animales, en que se inyectaba localmente PGs, observándose un aumento del número de osteoclastos, estimulando de este modo la reabsorción, acelerando finalmente el desplazamiento de la pieza $(8,9)$, la desventaja asociada a este procedimiento era la hiperalgesia en la zona.

Otro tipo de fármaco estudiado, es la osteocalcina, una de las principales proteínas no colágenas de la matriz ósea, que al ser administrada localmente estimula la aparición de osteoclastos en la superficie ósea del lado de presión durante el MDO en ratas, resultando esto en un aumento del movimiento dentario $(28,29)$.

Como desventajas estas técnicas requieren del uso de inyecciones, incluso en más de una ocasión, con las molestias que implica esto para el paciente. Se requieren más estudios para que estos avances puedan ser aplicados clínicamente.

\section{Agentes físicos}

Se han buscado modalidades menos invasivas para estimular el MDO, utilizando agentes físicos, como estimulaciones eléctricas, aplicación de láser y ultrasonido, que ya se han desarrollado en otras áreas de la medicina y odontología.

Existen reportes del uso experimental de corrientes eléctricas continuas de baja intensidad. Estudios realizados en animales y humanos indican que cuando se aplica este tipo de corriente al hueso alveolar, se modifica el potencial bioeléctrico, y el diente se mueve más rápido que su control en respuesta a un resorte idéntico $(2,30)$, pero se presentaron algunos inconvenientes que generaron el interés en obtener respuestas similares, con métodos menos invasivos. Así se comenzó a estudiar el efecto de los campos electromagnéticos, en el rango de movimiento dentario, estableciéndose un efecto facilitador del MDO por parte de los campos electromagnéticos pulsantes. Se obtuvieron también resultados histológicos que establecían un aumento de la actividad osteoblástica y de una mejor calidad de hueso en las zonas sometidas a tratamiento con campos electro- magnéticos (31). Otro estudio, realizado por Tengku y cols. (32), en ratas, incorporó campos electromagnéticos a los aparatos ortodóncicos, sin obtener un incremento en el MDO, pero observaron un aumento en la reabsorción radicular.

Otros estudios, como el realizado por Saito y Shimizu (33), analizaron el efecto del láser de baja intensidad, al ser aplicado en la sutura media, durante el estudio experimental de la expansión maxilar en ratas. Ellos determinaron que mediante la estimulación temprana con el láser se puede acelerar el proceso de regeneración ósea, disminuyendo el tiempo necesario de retención. Basado en este, y otros estudios, Cruz y cols. (27), estudiaron el efecto de la terapia con láser de baja intensidad en la velocidad del MDO en humanos, mostrando que la aplicación del láser de baja intensidad acelera significativamente la velocidad del MDO, debido a una estimulación de formación ósea es más rápida. A pesar del avance logrado en esta línea de investigación, aun no se conoce el mecanismo de acción del láser en la fisiología ósea y los parámetros de tratamientos requieren más estudios clínicos.

Otro agente físico que podría estimular el MDO es el ultrasonido pulsátil de baja intensidad (Low intensity pulsed ultrasound, LIPUS). En los últimos años se han realizado diversos estudios tanto en medicina como en odontología sobre el efecto del LIPUS en el tejido óseo, algunos de los hallazgos es que LIPUS ha demostrado ser efectivo en la liberación de factor de crecimiento fibroblástico desde células tipo macrófagos de la línea U937, además estimula la angiogénesis durante la cicatrización de heridas (34), aumenta el crecimiento óseo en los implantes de titanio de revestimiento poroso con una óseo integración más rápida (35), aumenta la reparación ósea después fracturas (36, 37) y en osteodistracción mandibular $(38,39)$.

El-Bialy y cols. (40), estudiando el efecto del ultrasonido en la formación y erupción de incisivos mandibulares de conejos luego de osteodistracción mandibular con una aplicación de 20 minutos diarios por cuatro semanas, encontraron que el LIPUS aumenta el crecimiento apical de los incisivos mandibulares y acelera su erupción, induciendo el crecimiento de tejido dentario y de soporte como dentina, cemento y tejido óseo. En un estudio realizado en babuinos 
(Papio hamadryas hamadryas), también realizado por El-Bialy y cols. (41), se observó un aumento del crecimiento mandibular al aplicar LIPUS sobre todo al combinarlo con aparatos funcionales. En el estudio de Harle y cols. (42), encontraron que el ultrasonido terapéutico estimula la expresión de proteínas óseas como osteonectina, osteopontina y sialoproteína ósea, siendo esta estimulación dosis dependiente. Según Nakago-Matsuo (43) y Graber (44), el LIPUS, como presiones hidráulicas, puede estimular la acción del oxido nítrico y L-arginina, generando una reacción tisular metabólica que aumenta las defensas del organismo en contra de una potencial secuela iatrogénica. En un estudio realizado por Shirazi y cols. (45), en que analizaron el rol del oxido nítrico en el movimiento dentario ortodóncico en ratas, encontrando que el aumento de oxido nítrico genera más remodelación ósea y mayor movimiento dentario ortodóncico, y disminuye las superficies de reabsorción, además aumenta la cantidad de osteoclastos alrededor de los dientes bajo movimiento ortodóncico. En un estudio más reciente de El-Bialy y cols. (44) realizado en humanos, aplicando LIPUS a premolares sometidos a fuerza ortodóncica que luego serían extraídos, encontraron una menor reabsorción radicular y demostró histológicamente que el proceso de reparación de la superficie radicular reabsorbida se produce por hipercementosis.

Con la evidencia encontrada en la literatura sobre el LIPUS, se podría esperar que al asociar una corriente ultrasónica pulsátil como el LIPUS al movimiento ortodóncico dentario, se pudiera obtener una remodelación ósea más rápida con una disminución en la reabsorción radicular, obteniendo un movimiento dentario ortodóncico más eficiente y con menos secuelas iatrogénicas para el paciente.

\section{CONCLUSIONES}

Las bases biológicas del MDO están dadas por las reacciones inflamatorias que ocurren en los tejidos paradentales, asociadas al proceso de remodelado óseo que se desencadena en respuesta a las fuerzas mecánicas aplicadas. Sin embargo, por la gran variedad de tejidos y células involucradas en estos procesos existe una gran cantidad de factores que pueden interferir en el remodelado óseo, alterando el rango y velocidad de MDO esperado. El efecto de las fuerzas mecánicas combinado con uno o más factores o agentes externos puede resultar inhibitorio, aditivo o sinérgico.

El ortodoncista debe estar consciente que el tratamiento ortodóncico se realiza en un tejido activo y reactivo, no en un modelo estático como un tipodonto, las respuesta que desencadenan las fuerzas ortodóncicas se pueden sobreponer con otros eventos que ocurren en cualquier parte del organismo, con alteraciones metabólicas del paciente, o fármacos que consuma el paciente en forma regular, que podrían alcanzar el tejido periodontal mecánicamente estresado, a través de la circulación sanguínea, interfiriendo con la tasa de remodelado óseo. Hoy en día, esto cobra mayor importancia debido al aumento de la consulta ortodóncica por parte del pacientes adultos, quienes poseen un historial médico y farmacológico más extenso, además de una mayor probabilidad de estar consumiendo algún tipo de fármaco, por lo cual, cada paciente debe ser explorado para determinar su potencial influencia en la mecanoterapia ortodóncica.

La creciente comprensión de la naturaleza biológica de los efectos de la carga mecánica sobre los tejidos y las células no sólo ha hecho que se tomen en cuenta los factores propios de paciente, sino que también a llevado a desarrollar experimentos destinados a encontrar los medios de alterar el MDO, de manera de facilitar el desarrollo de las mecánicas ortodóncicas. Todos estos estudios se basan en que las células son capaces de responder a una amplia gama de estímulos químicos, como agentes farmacéutico, y físicos, como campos magnéticos o corrientes eléctricas, logrando alterar el ritmo de remodelado óseo. No obstante, se continúan realizando estudios experimentales y clínicos al respecto, para desarrollar métodos de aplicación de estos agentes de manera sencilla y poco invasiva para el paciente para lograr un MDO más eficiente y con menos secuelas para los tejidos dentarios y periodontales.

\section{BIBLIOGRAFÍA}

1. Misawa-Kageyama Y, Kageyama T, Moriyama K, Kurihara S, Yagasaki H, Deguchi T, y cols. 
Histomorphometric study on the effects of age on orthodontic tooth movement and alveolar turn over in rats. Eur J Oral Sci 2007;115:12430.

2. Proffit WR. Biologic basis of orthodontic therapy. En Proffit WR, Fields HW. Contemporary Orthodontics. Mosby: St Louis; 2000.

3. Ong MA, Wang HL, Smith F. Interrelationship between periodontics and adults orthodontics. J Clin Periodontol 1998;25:271-7.

4. Ren Y, Maltha JC, Kuijpers-Jagtman AM, Van't Hof MA. Age effect on orthodontic tooth movement in rats. J Dent Res 2003;82:38-42.

5. Vellini F. Ortodoncia, Diagnóstico y Planificación Clínica. Artes Médicas Latinoamericanas: Sao Paulo;2002.

6. Canut J. Ortodoncia Clínica y Terapéutica. Masson: Barcelona; 2000.

7. Ong MM, Wang HL. Peridontics and orthodontics in adults. Am J Orthod Dentofacial Orthop 2002; 122:420-8.

8. Krishnan V, Davidovitch Z. The effect of drugs on orthodontic tooth movement. Orthod Craniofac Res 2006;9:163-71.

9. Tyrovola JB, Spyropoulos MN. Effects of drug and systemic factors in orthodontics treatment. Quintessence Int 2001;32:365-71.

10. Arias O, Marquez-Orozco M. Aspirin, acetaminophen, and ibuprofen: Their effects on orthodontic tooth movement. Am J Orthod Dentofacial Orthop 2006;130:364-70.

11. Sámano R, Lezama D. Influencia de las Prostaglandinas en el movimiento dental ortodóncico. Rev ADM 1999;56:59-63.

12. Gameiro GH, Pereira-Neto JS, Magnani MB, Nouer DF. The influence of drugs and systemic factor son orthodonticc tooth movement. J Clin Orthod 2007;41:73-8.
13. Wong A, Reynolds EC, West VC. The effect of acetylsalicylic acid on orthodontic tooth movement in the guinea pig. Am J Orthod Dentofacial Orthop 1992;102:360-5.

14. Gameiro GH, Nouer DF, Pereira-Neto JS, Siqueira VC, Andrade ED, Novaes PD, y cols. Effects of short- and long-term celecoxib on orthodontic tooth movement. Angle Orthod 2008;78:860-5.

15. Sari E, Olmez H, Gürton AU. Comparison of some effects of acetylsalicylic acid and rofecoxib during orthodontic tooth movement. Am J Orthod Dentofacial Orthop 2004;125:310-5.

16. Lucas R, Warner TD, Vojnovic I, Mitchell JA. Cellular mechanism of acetaminophen: role of cyclo-oxygenase. FASEB J 2005;19:635-7.

17. Roche JJ, Cisneros GJ, Acs G. The effect of acetaminophen on tooth movement in rabbits. Angle Orthod 1997;67:231-6.

18. Ashcraft MB, Southard KA, Tolley EA. The effect of corticosteroid-induced osteoporosis on orthodontic tooth movevement. Am J Orthod Dentofacial Orthop 1992; 102:310-9.

19. Bartzela T, Türp JC, Motschall E, Maltha JC. Medications effect on the rate of orthodontic tooth movement: A systematic literature review. Am J Orthod Dentofacial Orthop 2009;135:16-26.

20. Kalia S, Melsen B, Verna C. Tissue reaction to orthodontic tooth movement in acute and chronic corticosteroid treatment. Orthod Craniofac Res 2004;7:26-34.

21. Graham JW. Bisphosphonates and Orthodontics: Clinical Implications. J Clin Orthod 2006;40: 425-8.

22. Zahrowski JJ. Biphosphonate treatment: an Orthodontic concern calling for proactive approach. Am J Orthod Dentofacial Orthop 2007;131:311-20.

23. Igarashi K, Adachi $\mathrm{H}$, Mitani $\mathrm{H}$, Shinoda $\mathrm{H}$. Inhibitory effect of topical administration of 
bisphosphonate (Risedronate) on root resorption incident to orthodontic tooth movement in rats. J Dent Res 1996;75:1644-9.

24. Dolce C, Vakani A, Archer L, Morris-Wiman JA, Holliday LS. Effects of echistatin and RGD peptide on orthodontics tooth movements. J Dent Res 2003;82:682-6.

25. Kanzaki H, Chiba M, Shimizu Y, Mitani H. Periodontal ligament cells under mechanical stress induce osteoclastogenesis by receptor activator of nuclear factor kappa B ligand upregulation via prostaglandin E2 synthesis. J Bone Miner Res 2002; 17:210-20.

26. Kanzaki H, Chiba M, Takahashi I, Haruyama N, Nishimura M, Mitani H. Local OPG gene transfer to periodontal tissue inhibits orthodontics tooth movements. J Dent Res 2004;83:920-5.

27. Cruz DR, Kohara EK, Ribeiro MS, Wetter NU. Effects of low-intensity laser therapy on the orthodontic movement velocity of human teeth: a preliminary study. Lasers Surg Med 2004;35:117-20.

28. Hashimoto F, Kobayashi Y, Mataki S, Kobayashi K, Kato Y, Sakai H. Administration of osteocalcin accelerates orthodontic tooth movement induced by closed coil spring in rats. Eur J Orthod 2001;23:535-45.

29. Kobayashi Y, Takagi H, Sakai H, Hashimoto F, Mataki S, Kobayashi K, y cols. Effects of local administration of osteocalcin on experimental tooth movement. Angle Orthod 1998;68:259-66.

30. Davidovitch Z, Finkelson MD, Steigman S, Shanfeld J, Montgomery P, Korostoff E. Electric currents, bone remodeling and orthodontic tooth movement. I. The effect of electric currents on periodontal cyclic nucleotides. Am J Orthod 1980;77:14-32.

31. Fraticelli D, Gandini P, Sfondrini MF, Botticelli A, Zaffe D. Tratamiento ortodóncico y campos electromagnéticos pulsantes. Rev Esp Ortod 1998; 28:55-61.
32. Tengku BS, Joseph BK, Harbrow D, Taverne AA, Symons AL. Effect of static magnetic field on orthodontic tooth movement in rat. Eur $\mathrm{J}$ Orthod 2000;22:475-87.

33. Saito S, Shimizu N. Stimulatory effects of lowpower laser irradiation on bone regeneration in midpalatal suture during expansion in rats. Am J Orthod Dentofacial Orthop 1997;111:525-32.

34. Young SR, Dyson M. The effect of therapeutic ultrasound on angiogenesis. Ultrasound Med Biol 1990;16:261-9.

35. Tanzer M, Harvey E, Kay A, Morton P, Bobyn JD. Effect of noninvasive low intensity ultrasound on bone growth into porous-coated implants. J Orthop Res 1996; 14:901-6.

36. Abramovich A. Effect of ultrasound on the tibia of the young rat. J Dent Res 1970;49:1182.

37. Heckman JD, Ryaby JP, McCabe J, Frey JJ, Kilcoyne RF. Acceleration of tibial fracture-healing by non-invasive, low-intensity pulsed ultrasound. J Bone Joint Surg Am 1994;76:26-34.

38. Shimazaki A, Inui $\mathrm{K}$, Azuma $\mathrm{Y}$, Nishimura $\mathrm{N}$, Yamano Y. Low-intensity pulsed ultrasound accelerates bone maturation in distraction osteogenesis in rabbits. J Bone Joint Surg $\mathrm{Br}$ 2000 82:1077-82.

39. El-Bialy TH, Royston TJ, Magin RL, Evans CA, Zaki Ael-M, Frizzell LA. The effect of pulsed ultrasound on mandibular distraction. Ann Biomed Eng 2002; 30:1251-61.

40. El-Bialy TH, Zaki AE, Evans CA. Effect of ultrasound on rabbit mandibular incisor formation and eruption after mandibular osteodistraction. Am J Orthod Dentofacial Orthop 2003;124:427-34.

41. El-Bialy T, Hassan A, Albaghdadi T, Fouad HA, Maimani AR. Growth modification of the mandible with ultrasound in baboons: A preliminary report. Am J Orthod Dentofacial Orthop 2006;130: 435.e7-14. 
42. Harle J, Salih V, Mayia F, Knowles JC, Olsen I. Effects of ultrasound on the growth and function of bone and periodontal ligament cells in vitro. Ultrasound Med Biol 2001;27:579-86.

43. Nakago-Matsuo C, Matsuo T, Nakago T. Basal nitric oxide production is enhanced by hydraulic pressure in cultured human periodontal ligament fibroblasts. Am J Orthod Dentofacial Orthop 2000;117:474-8.

44. El-Bialy TH, El-Shamy I, Graber TM. Repair of orthodontically induced root resorption by ultrasound in humans. Am J Orthod Dentofacial Orthop 2004;126:186-93.

45. Shirazi M, Niforoushan D, Alghasi H, Dehpour R. The role of nitric oxide in orthodontic tooth movement in rats. Angle Orthod 2002;72:211-5.

\section{CORRESPONDENCIA}

Paula Vargas del Valle E-mail:paulavdv@gmail.com

Becas o ayudas: Proyecto de investigación DI 01-08/ CB 